\title{
Analysis of the Range of Acceleration for an Accelerometer with Extended Beams
}

\author{
Margarita Tecpoyotl-Torres ${ }^{1}$, Ramon Cabello-Ruiz ${ }^{1}$, Jose Gerardo Vera-Dimas ${ }^{1}$, \\ Alfonso Torres-Jacome ${ }^{2}$, Pedro Vargas ${ }^{1}$, Svetlana Koshevaya ${ }^{1}$ \\ ${ }^{1}$ Centro de Investigacion en Ingenieria y Ciencias Aplicadas-(IICBA), Universidad Autonoma del Estado de Morelos \\ ${ }^{2}$ Instituto Nacional de Astrofisica, Optica y Electronica
}

\begin{tabular}{l} 
Article Info \\
\hline Article history: \\
Received Jan 19, 2016 \\
Revised Apr 15, 2016 \\
Accepted Apr 29, 2016 \\
\hline
\end{tabular}

Keyword:

Accelerometer

Gravity

MEMS

Normal stress

\begin{abstract}
The elastic behaviour of a system can be determined by an analysis of stresses. The stress generated in the element loaded of an accelerometer is of interest here. In these devices, the suspension beams are the elements subjected to greater stresses, as they support the mass. The stress that they can support is limited by the elastic limit of the material. Based on this analysis, the operating conditions to prevent permanent deformations are determined. The analysis is focused on the acceleration applied to the accelerometer because this parameter increases considerably the stresses in the device. A relationship between normal stress and gravity applied is obtained. This equation is used in order to avoid exceeding the elastic limit, during the accelerometer operation. This fact determines the acceleration range supported by the device. In the literature, studies about the physics and modelling of accelerometers are performed. However, about the specific acceleration of operation which they are subjected, information about its determination is not provided. In this paper, the analysis is realized considering a Conventional Capacitive Accelerometer (CCA) and a Capacitive Accelerometer with Extended Beams (CAEB), particularly, on the normal stress. When a range of acceleration values are applied, normal stress occur which must not exceed the elastic limit of the material, as it was mentioned before. The Matlab code used to calculate this relationship is given in Appendix A.
\end{abstract}

Copyright (C) 2016 Institute of Advanced Engineering and Science. All rights reserved.

\section{Corresponding Author:}

Margarita Tecpoyotl-Torres,

Centro de Investigacion en Ingenieria y Ciencias Aplicadas-(IICBA),

Universidad Autonoma del Estado de Morelos,

Av. Universidad 1001. 62209 Cuernavaca, Morelos, Mexico.

Email: tecpoyotl@uaem.mx

\section{INTRODUCTION}

An accelerometer is a sensor device that allows the indirect measurement of acceleration (speed variation with time or relation between force and mass) according to one, two, or three directions throughout a sensible axis [1]. Accelerometers have exploited their applications in various fields like monitoring vibration, inertial navigation, and attitude controlling. The most common accelerometers are conventionally based on capacitive, piezoresistive or piezoelectric behavior [2],[3]. To extract the acceleration value, the sensor has a movable proof mass connected to a fixed frame through spring structures. When there is an external acceleration, the seismic mass is displaced from its rest position. The magnitude of this displacement is proportional to the magnitude of the acceleration and inversely proportional to the stiffness of the spring structures [4].

The accelerometers are constituted by structural supports called suspension beams. It is important to realize an analysis of stress into them, in order to obtain the extreme operating conditions. In this paper, the 
acceleration range in which an accelerometer with extended suspension beams can operate appropriately is presented. For this reason, the analysis of normal stress in the suspension beams is realized, using the model of a cantilever beam with a uniformly distributed load. Subsequently, the information obtained is validated using Ansys.

\subsection{Analytic Relation Normal Stress-Acceleration}

In capacitive accelerometers, the structural elements of support are called beams. They allow the mass to be suspended and displaced. Their study is fundamental to understand the analysis of normal stress.

Due to the applied loads, the beams develop a shear force and a bending moment that, in general, change from point to point along the axis of the beams [5]. The beams can be classified according to their conditions of support, as follows: a) simply supported beams. The reactions happen in their ends, b) cantilever. One end of the beam is fixed to prevent rotation, c) cantilevered beams. One or both ends of them stands out of the supports, d) constant beams. A statically indeterminate beam that spreads on three or more supports.

The load consists on the applied forces that act on the beam, which can come from the weight of the beam, besides other forces that it could support. There are five basic types of loads applied in beams, which are: a) without load. The same beam is considered without weight (or at least very small compared with other forces that could be applied), b) concentrated load. A load applied on a relatively small area, c) uniformly distributed loads. This load is equally distributed on a portion of length of beams, d) variable load. The load varies its intensity form of one place to other, e) torsion. This one is generated when a torsion is applied on any part of the beam [6].

For the accelerometers used here, the type of beams are cantilevers and the considered load is uniformly distributed. The bending moment $M$, where the normal stress remains below the yield strength or elastic limit $\sigma_{y}$, is also studied, because it serves as reference parameter. The stress in cantilevers must be remained below the elastic limit so there will not be permanent deformations. The Hooke's law can be applied to the stress uniaxial calculation.

Assuming a homogeneous material, and denoting for $E$ to the modulus of elasticity, on the longitudinal direction $\mathrm{x}$, the stress is given by:

$$
\sigma_{x}=E \varepsilon_{x}
$$

where $\varepsilon_{x}$ is unitary longitudinal deformation, calculated from $\varepsilon_{x}=-\frac{y}{c} \varepsilon_{m}, c$ is the maximum distance to the neutral surface, $\mathrm{y}$ is the distance of the neutral axis to any point of beams and $\varepsilon_{m}$ is the maximum absolute value of the unitary deformation. Multiplying both members of (1) by $E$, the normal stress can be obtained:

$$
\sigma_{x}=-\frac{y}{c} \sigma_{m}
$$

where $\sigma_{m}$ is the maximum absolute value stress. This result shows that, in the elastic range, the normal stress changes linearly with the distance to the neutral axis (Figure 1) [7].

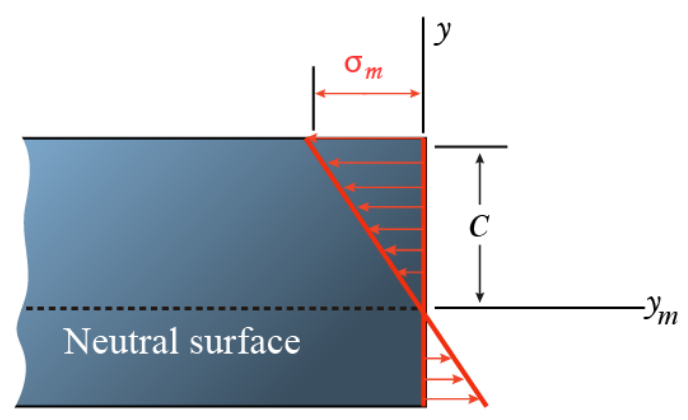

Figure 1. Variation of the normal stress [7] 
To calculate $\sigma_{m}$ under the case of pure bending, where the neutral axis passes for the centroid section, the inertia moment $I$, or the second moment of the cross section with respect to the centroid axis perpendicularly to the plane of the par $M[6]$, is also considered:

$$
\sigma_{m}=\frac{M \cdot c}{I}
$$

Replacing $\sigma_{m}$ from (3) in (2), the normal stress $\sigma_{x}$ to any distance $y$ of the neutral axis is obtained:

$$
\sigma_{x}=\frac{M \cdot y}{I}
$$

In addition, $I$ is calculated by means of (5) where $b$ is the thickness and $h$ is the width of the beam.

$$
I=\frac{b h^{3}}{12}
$$

If there is not exist a load concentrated in the free end of the cantilever, the unique load that it experiences is only the produced by its own weight. Then the load is distributed in a uniform way, as Figure 2 shows [8].

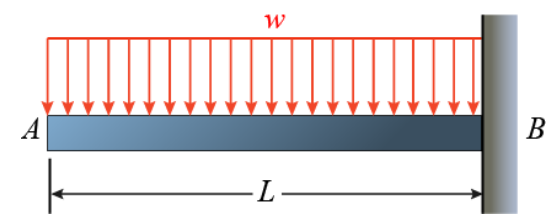

Figure 2. Cantilever with uniformly distributed load [7]

To calculate the bending moment of a cantilever with uniformly distributed load, the following equation is used:

$$
M=\frac{1}{2} w L^{2}
$$

where $L$ is the length of the cantilever and $w$ is the load distributed by unit of length:

$$
w=\frac{m \cdot g}{L}
$$

The type of beam and load considered in this work are showed in Figure 2. Replacing (7) in (6), following expression is obtained:

$$
M=\frac{1}{2} m \cdot g \cdot L
$$

To obtain the relationship between normal stress and the bending moment that will determine the rate of acceleration of the accelerometer, (8) is replaced in (4). Finally, the desired relationship between normal stress and gravity, is obtained:

$$
\sigma_{x}=\frac{m \cdot g \cdot L \cdot y}{2 \cdot I}
$$

It is noteworthy that in the analyzed literature this relationship is not developed or shown. 


\section{RESEARCH METHOD}

\subsection{Theoretical analysis and simulation of normal stress in a conventional accelerometer at $\mathbf{1 g}$}

The accelerometers are devices used to measure acceleration and vibration. These devices convert the acceleration of the gravity or of the movement into an electrical analogical signal, proportional to the force applied to the system [9]. Figure 3 shows the main elements of a CCA.

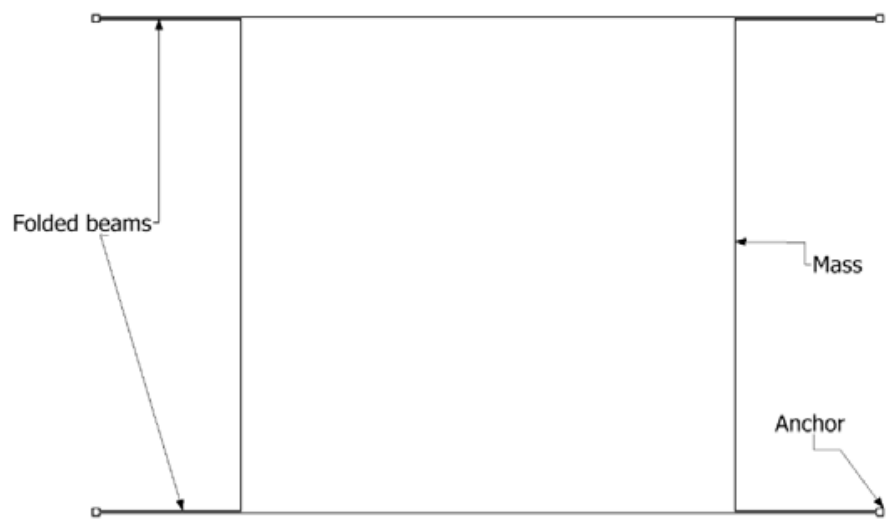

Figure 3. Main elements of the CCA

The calculation of normal stress $\sigma_{x}$ is made from (9). The corresponding values are shown in Table 1.

Table 1. Calculated parameter values

\begin{tabular}{cc}
\hline Parameter & Value \\
\hline Load, $w$ & $0.014 \mathrm{~N} / \mathrm{m}$ \\
Bending moment, $M$ & $2.81 \times 10^{-8} \mathrm{~N} \cdot \mathrm{m}$ \\
Inertia moment, $I$ & $3.25 \times 10^{-20} \mathrm{~m}^{4}$ \\
Normal stress, $\sigma_{x}$ & $10.8 \mathrm{MPa}$ \\
\hline
\end{tabular}

In order to compare the approximation to calculate the normal stress, given by (9), the simulation was implemented. Applying $1 g(9.81 \mathrm{~m} / \mathrm{s} 2)$ to CCA. A $2.59 \mathrm{MPa}$ normal stress value was obtained. As it can be observed, the theoretical value appears to be larger than the one obtained by simulation. This is because the calculation is performed for only one suspension beam. To obtain the normal stress value it is necessary to divide the total value between the number of beams. The result is $2.7 \mathrm{MPa}$ for each one of the suspension beams. The difference produces an error of $4 \%$.

Table 2 shows the properties of silicon used in theoretical calculations and by simulation, in the development of this work.

Table 2. Silicon properties used [10]

\begin{tabular}{cc}
\hline Property & Value \\
\hline Desnsity $(\rho)$, in $\mathrm{kg} / \mathrm{m}^{3}$ & 2330 \\
Young's Modulus $(E)$, in GPa & 131 \\
Poisson's ratio, dimensionless & 0.33 \\
\hline
\end{tabular}

Figure 4 shows the considered dimensions for the CCA. 


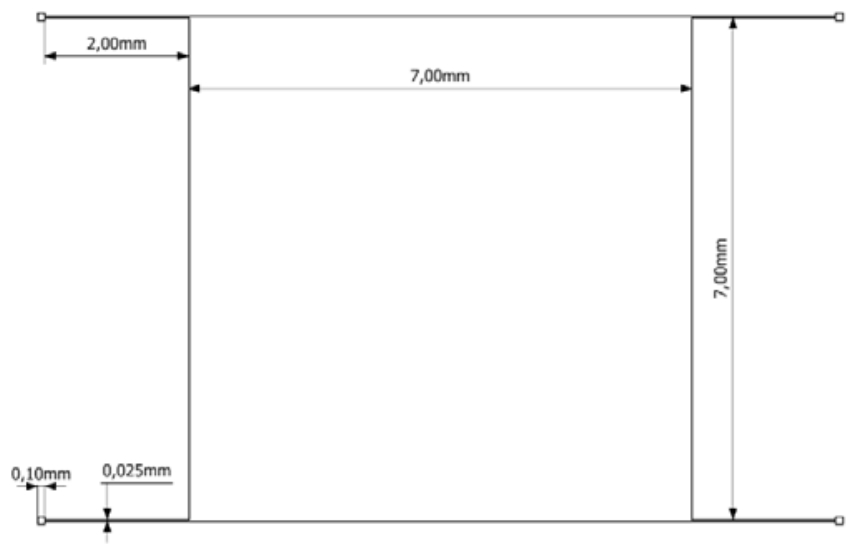

Figure 4. Dimensions of the CCA

In Figure 5 and 6, the normal stress generated in the suspension beams and a zoom in at one of them are shown, respectively.

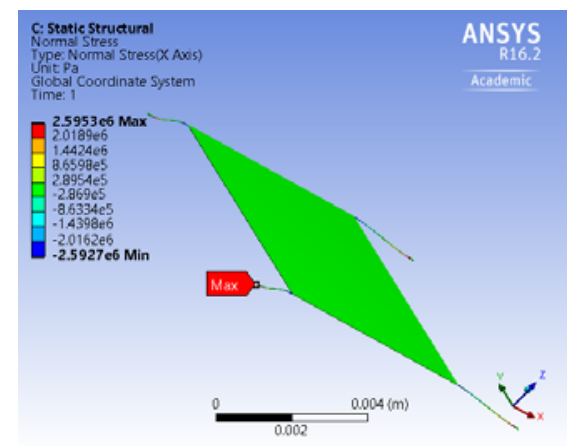

Figure 5. Normal stress generated in the suspension beams of a CCA

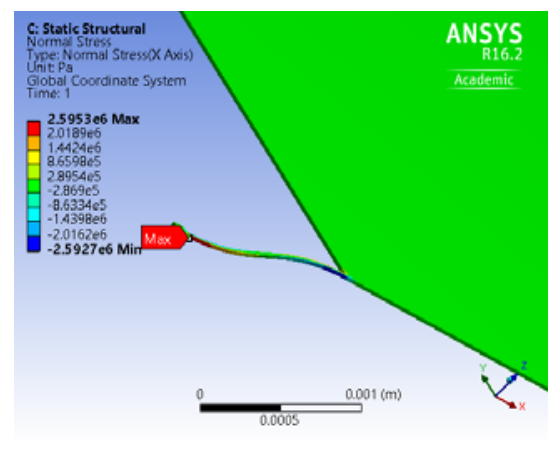

Figure 6. Zoom in at one of the folded beam

\subsection{Theoretical analysis and simulation of normal stress in a CAEB at $1 \mathrm{~g}$}

Once the calculations have been made for a CCA subjected at $1 g$, the case of the CAEB will be analysed. Figure 7 shows the dimensions of this accelerometer, generated from a change in the geometry of the conventional mass, intended to extend the length of the suspension beams, without excessively reduce the value of the mass, as it is required by equation of sensitivity, given by (10):

$$
x=\frac{m \cdot g}{k}
$$

where $x$ is the sensitivity displacement and $k$ is the stiffness constant.

However, the increment on beams length implies greater bending moment (6), which also implies an increase of normal stress (9). Then, the normal stress limits the beams extension and the acceleration value. The analysis in this case is similar to the one realized in section 2.1. In Table 3, the calculated values are presented.

Table 3. Calculated parameter values

\begin{tabular}{cc}
\hline Parameter & Value \\
\hline Load, $w$ & $0.005 \mathrm{~N} / \mathrm{m}$ \\
Bending moment, $M$ & $5.43 \times 10^{-8} \mathrm{~N} \cdot \mathrm{m}$ \\
Inertia moment, $I$ & $3.25 \times 10^{-20} \mathrm{~m}^{4}$ \\
Normal stress, $\sigma_{x}$ & $20.8 \mathrm{MPa}$
\end{tabular}

Analysis of the Range of Acceleration for an Accelerometer with Extended Beams (Margarita Tecpoyotl T.) 
Figure 7 shows the dimensions of a CAEB.

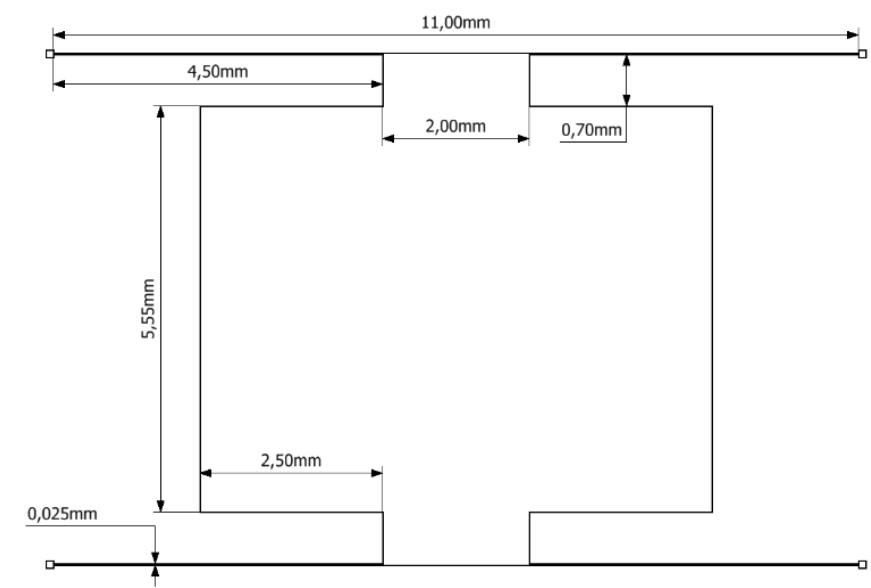

Figure 7. Dimensions of an accelerometer with extended beams

Figure 8 shows the normal stress generated in the suspension beams, and in Figure 9 a zoom in at one of the suspension beam is shown.

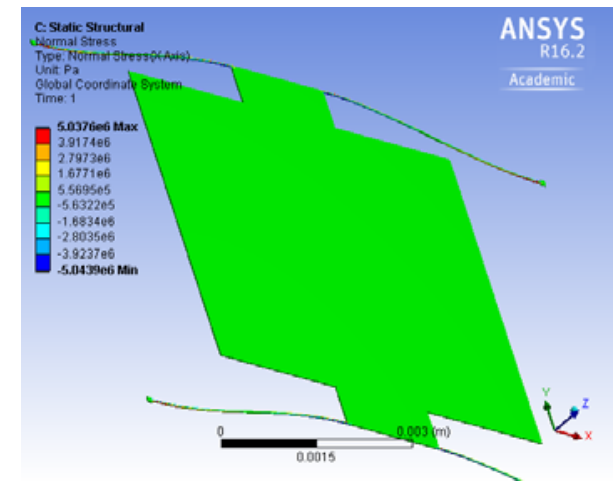

Figure 8. Normal stress generated in the suspension beams of a CAEB

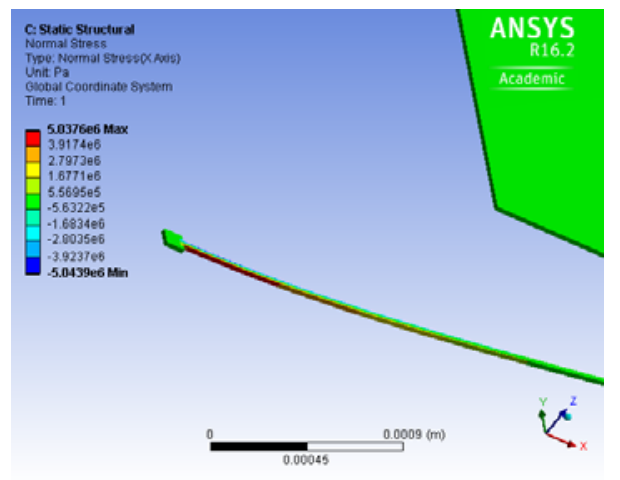

Figure 9. Zoom in at one of the folded beam

From Figure 9, the simulation results provide a normal stress value of 5.03 $\mathrm{MPa}$. In a similar fashion to the procedure followed in the case of the CCA, it is necessary to divide this total value by the number of suspension beams. 5.2 MPa normal stress value was obtained, which is very close to 5.03 MPa obtained from the simulation, throwing an error of $3 \%$. As it is shown, the change in geometry of the mass determines a more precise analytical calculation. The increment in the arm length implies an increase in the normal stress, because an increased bending moment occurs.

\section{RESULTS AND ANALYSIS}

In this section, the theoretical and simulated results of the accelerometers are presented, performing a sweep in the range of the applied acceleration. As it is shown, from simulation results of Sections 2.1 and 2.2, the analytical approach is useful for stress calculation. Since the normal stress generated in the suspension beams does not exceed the elastic limit of silicon $(250 \mathrm{MPa})$ when $1 \mathrm{~g}$ is applied to the accelerometers shown previously (section 2.1 and 2.2), we proceed to realize an analysis with larger values of $g$. Figure 10 shows the normal stress generated in the suspension beams of CCA, when $97 g^{\prime} s$ are applied. 
The value of the normal stress generated in the suspension beams is $251 \mathrm{MPa}$ at $97 \mathrm{~g}^{\prime} s$ (Figure 10). It indicates that the elastic limit has been exceeded, which may indicate that this accelerometer, fabricated in silicon, can operate properly until $96 \mathrm{~g}$ 's approximately, where the normal stress value is of $249.149 \mathrm{MPa}$. In Figure 11, the normal stresses values obtained theoretically and by simulation are shown, from $1 g$ to $96 g^{\prime} s$ for CCA. To ensure the integrity of the device we suggest as operating limit up to $92 g^{\prime} s$.

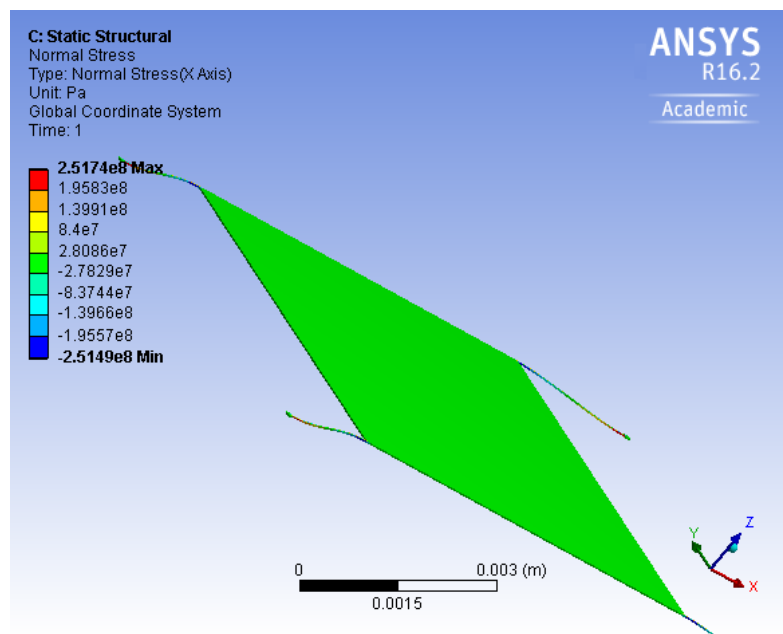

Figure 10. Normal stress generated in suspension beams of the conventional accelerometer, when $97 \mathrm{~g}$ 's are applied

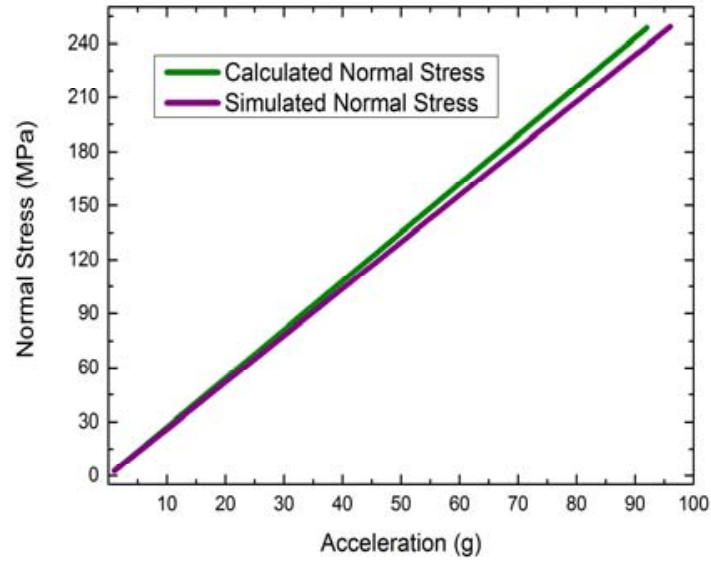

Figure 11. Calculated and simulated normal stress generated in suspension beams of a CCA

Figure 12 shows the normal stress generated in the suspension beams of the CAEB, shown in section 2.2, with $50 g^{\prime} s$ applied.

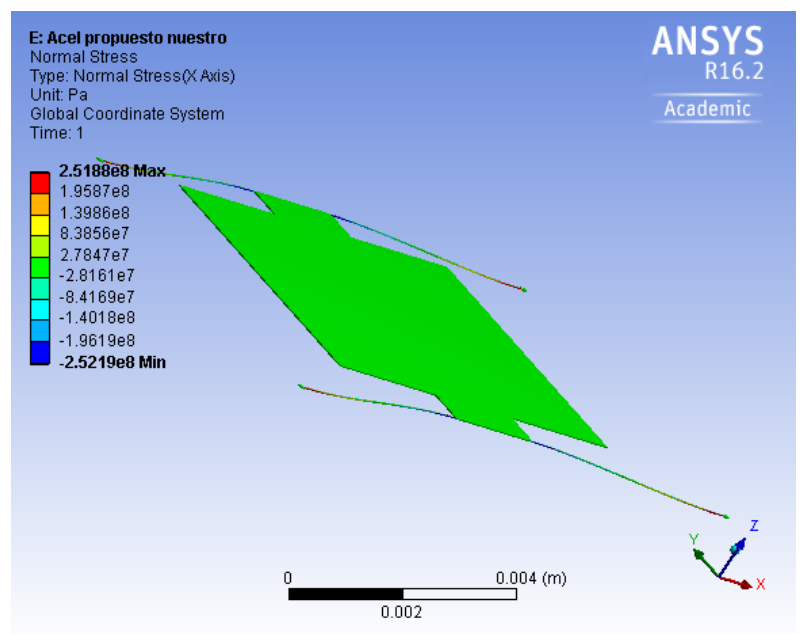

Figure 12. Normal stress generated in suspension beams of CAEB, with $50 g^{\prime} s$ applied

Figure 12 shows how the elastic limit of the silicon is exceeded. $251 \mathrm{MPa}$ are obtained at $50 \mathrm{~g}^{\prime} s$. Figure 13 shows the normal stress obtained theoretically and by simulation, from $1 g$ up to $50 g^{\prime} s$. At $49 g^{\prime} s$ the value of the normal stress is $246.84 \mathrm{MPa}$. Again, to ensure device integrity operating limit until $47 \mathrm{~g}^{\prime} s$ is suggested. 


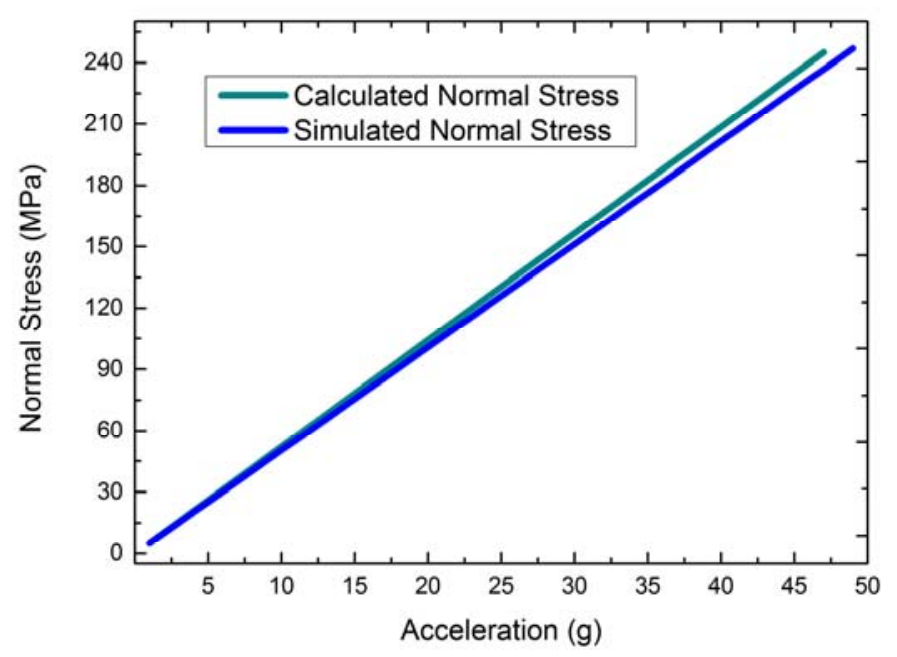

Figure 13. Calculated and simulated normal stress generated in suspension beams of a CAEB

\section{CONCLUSION}

The identification of the types of support of the suspension beams and of load are important in the theoretical analysis of normal stress. They have been identified as cantilever and uniformly distributed load, respectively. The error between simulated and calculated results of normal stress for both cases is small, $4 \%$ for CCA and 3\% for CAEB. This fact is due to the variables used in the calculation of the load.

The extension of the length of the beams produces considerably greater stresses. At $1 g$, normal stresses of $2.5 \mathrm{MPa}$ and $5.2 \mathrm{MPa}$ is generated for cases of CCA and of CAEB, respectively. While, the upper limit values are reached at accelerations of 96 and $47 \mathrm{~g}$ 's, producing simulated normal stresses near to the yield stress of $249 \mathrm{MPa}$, for the CCA and CAEB, respectively.

It can be observed an inverse relationship between the applied normal force and acceleration. That means, CCA supports a wider range of acceleration than CAEB. Therefore, CAEB is recommended for systems requiring low levels of acceleration. Knowledge of the relationship between normal stress and acceleration is fundamental in the calculation of the range of acceleration supported by the accelerometer. The parameters that influence this relationship are of physical and geometric nature. The calculation procedure is given in this work and summarized in (9).

\section{APPENDIX A}

clear all

In this appendix, we show the code generated en MATLAB.

close all

clc

$\operatorname{datax}=[]$

$\operatorname{data\mathrm {E}}=[]$;

$w b=25 * 10 \wedge-6$

$\mathrm{E}=131 * 10 \wedge 9$;

$\mathrm{h}=25 * 10 \wedge-6$;

$\mathrm{g}=9.81$;

pi=3.141592;

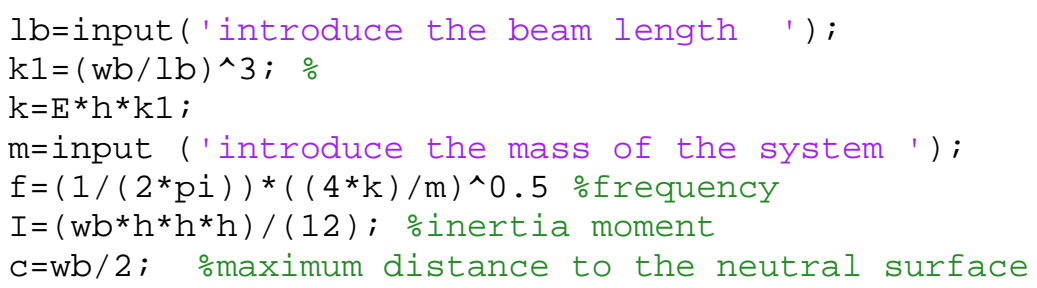




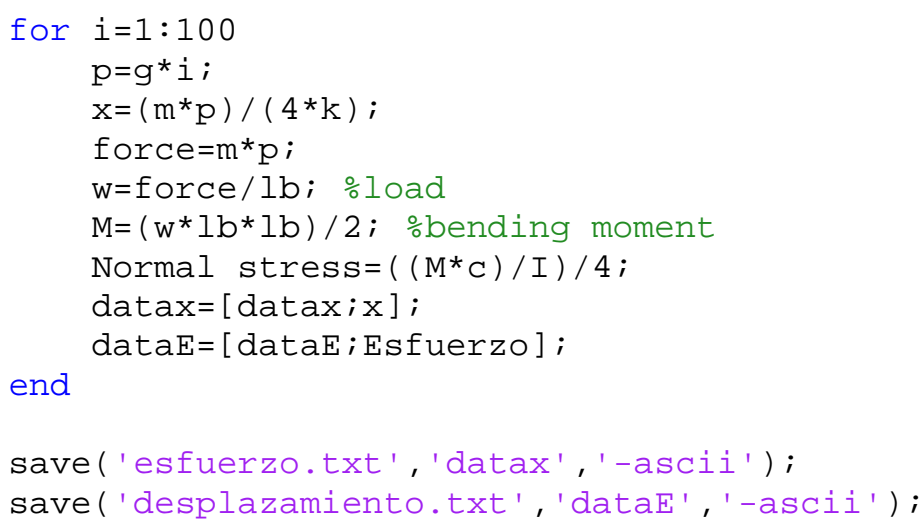

\section{ACKNOWLEDGEMENTS}

R Cabello and P Vargas expressed their sincere thanks to CONACYT for the scholarship with grants reference $376566 / 248576$ and $484392 / 273928$, respectively.

\section{REFERENCES}

[1] Albarbar A., "Performance evaluation of MEMS accelerometer Measurement," vol. 42, pp. 790-5, 2009.

[2] Macdonald G. A., "A review of low cost accelerometers for vehicle dynamics Sens," Actuators A, vol. 21, pp. 303307, 1990.

[3] Yazdi N., et al., "Micromachined inertial sensors," Proc. IEEE, vol. 86, pp. 1640-1659, 1998.

[4] B. Shafaat, et al., "Monolithic Tri-Axes Nickel-Based Accelerometer Design Verified Through Finite Element Analysis," Arab J Sci Eng, vol. 38, pp. 2103-2113, 2013.

[5] H. Jones R. C., "Mecanica de materiales," Ed. Pearson, 2006.

[6] Fitzgerald R. W., "Mecanica de materiales," Ed. Alfaomega, 2007.

[7] Beer F. P., et al., "Mecanica de materiales," Ed. McGraw-Hill, 2009.

[8] G. Cruz C. T., "Diseño de sensores basados en la tecnologia de microsistemas," Thesis, 2011.

[9] Manzanares A., "Estudio de modelos matematicos de acelerometros comerciales," Thesis, 2008.

[10] http://www.ansys.com/

\section{BIOGRAPHIES OF AUTHORS}

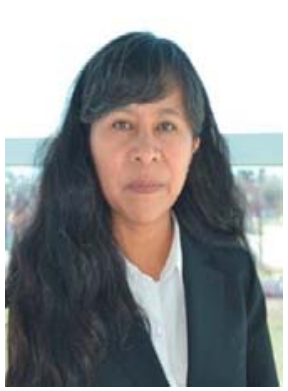

Margarita Tecpoyotl Torres received the Mathematician degree from the University of Puebla, Mexico, in 1991. From this University, she was also graduated as Electronic Engineer in 1993. She received the M.Sc. and Ph.D. degrees in Electronics from National Institute of Astrophysics, Optics and Electronics, INAOE, México, in 1997 and 1999, respectively. Dr. Tecpoyotl works, since 1999, at CIICAp of Autonomous University of Morelos, Mexico, where she is currently titular professor. She has been visiting research scientist in University of Bristol (2001), UK. She led the Winner team of Boot Camp, UAEM Potential obtaining support by TECHBA to go to SILICON VALLEY in May of 2014. In the same year, she was co-founder of INNTECVER. In 2015 she won the third place in the Royal Academy of Engineering's Leaders in Innovation Fellowships final pitch session, in UK. Her main research interest includes MEMS, Antenna design, Microwave devices, entrepreneurship and innovation; and also, development of educational programs. She has currently four patents titles from IMPI, two copyrights, and one trademark. She holds the status of National Researcher (SNI), in Mexico since 1999.

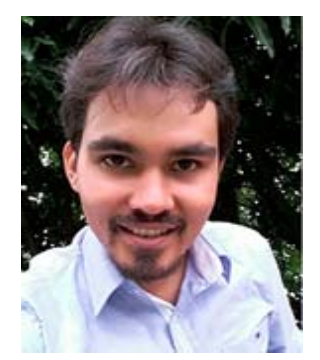

Ramon Cabello Ruiz received the degree of Mechanical Engineer (2010) from the Autonomous University of Morelos (UAEM), Mexico. And he studied the Mastery of Engineering in the Center for Applied Research in Engineering and Applied Sciences (CIICAp), Mexico, in 2012. Ramon Cabello is studying a PhD in Engineering in CIICAp Sciences, moreover, that has given classes at the School of Chemical Sciences and Engineering at the UAEM. 

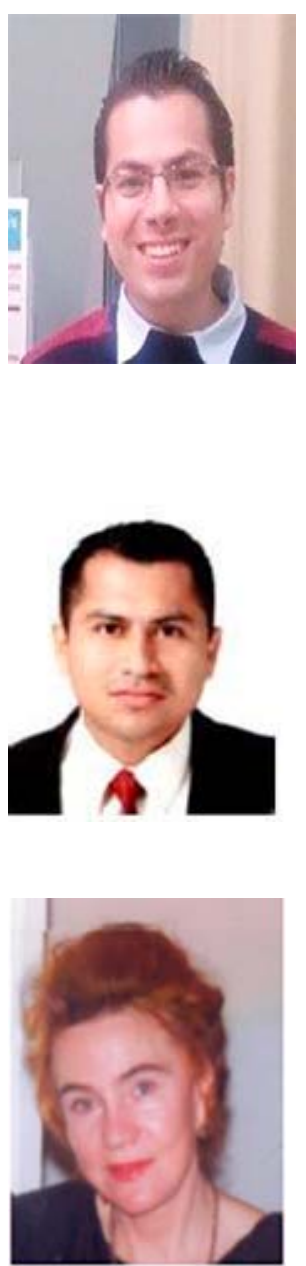

José Gerardo Vera is graduated from the Technological Institute of Morelia as Electronic Engineer. Currently, he is organizing member of ROPEC editions VII and VIII. He received the EGRETEC 2009 award as Young Graduated from the Graduated Association of the Technological Institute of Morelia. He got his Master degree with honors in the Research Center of Engineering and Applied Sciences (CIICAp) belonging to the Autonomous University of Morelos State (UAEM). He also got the PhD degree in Engineering and Applied Science in Electrical area. Gerardo Vera is part of the development of the curriculum of the Master in Commercialization of Innovative Knowledge and the Bachelor on Technology, both of CIICApUAEM. He was winner of the BootCamp 2013 organized by TechBA, also he is part of the winner team of a scholarship to be part of the program FULL IMMERSION in TechBA Silicon Valley.

Pedro Vargas Chablé Received the B. Sc. Degree by the Autonomous University Juarez from Tabasco in 2008. From 2009 to 2012, he was Technical Specialist Assesment of Lighting Conditions and Non-Ionizing Radiation, NOM-025-STPS-2008 and NOM-013-STPS-1993 respectively, in Environmental Technology S.A of C.V. In 2014 he Received M.Sc. Degree at the Autonomous University of Morelos State (UAEM). He is a PhD student at the Research Center on Engineering and Applied Science (CIICAp) of the UAEM. His current research interest are FEA, microgripper, microactuators and VLSI.

Svetlana Koshevaya received the Diploma of Master from Faculty of Radiophysics, Physical Electronics Dept., Kiev University, in 1964, the Ph. D. in Radiophysics from Kiev Institute of Radioproblems, Kiev University in 1969, and the diploma of Doctor of Science, from Kiev University, in 1986. Dra. Koshevaya worked as Engineer (1964-1968), in Kiev Institute of Radioproblems, Ukraine. She was Junior Senior Research Scientist Research Scientist (19681970) in Kiev Institute of Radioproblems and Senior Research Scientist in Institute "Orion" (1979-1972), Kiev, Ukraine. In Faculty of Radiophysics of Kiev National University, she was Senior Research Scientist (1972-1974), Principal Lecturer (1974-1980), Associate Professor (1980-1987) and Full Professor (1987 -1995). She was Titular Researcher "C" (1995- 1998), in INAOE, Puebla, Mexico. Since 1998, she is Titular Researcher "C" at CIICAp, Autonomous University of State Morelos (UAEM), Cuernavaca, Mexico. Her research interests include remote sensing system for seism and volcano activity, photonics and submillimeter wave integrated technique, nonlinear radiolocation, and solitonics in nonlinear physics. She has 7 books (in Russia), two chapters in books published in English, one book with student in Spanish, 15 certificate of patents, 196 Papers in international journals and 143 Articles in Proceedings of Symposiums . She is member of Mexican Academia of Science, member of National System of Researchers (SNI) and Member of WHO IS WHO 